\title{
An Interdomain MIH-FDMM Dynamic Anchoring Supports CVBR QoS in Mobile Internetworking
}

\author{
R. Tamijetchelvy ${ }^{1 *}$ and G. Sivaradje ${ }^{2}$ \\ ${ }^{1,2}$ Department of Electronics and Communication Engineering \\ ${ }^{1}$ Perunthalaivar Kamarajar Inst. of Engineering and Technology, India ${ }^{2}$ \\ Pondicherry Engineering College, India \\ ${ }^{1 *}$ saitamilselvi@yahoo.com, ${ }^{2}$ shivaradje@pec.edu
}

\begin{abstract}
t
The advent multimedia real time application demands large volume of data to execute the required quality of service $(Q o S)$. Existing centralized approach lacks support its suitability in terms of dynamic mobility, scalability, reliability, seamless connectivity, etc. This motivates host based dynamic and distributed mobility management (DMM) solution for MIPv6 protocol to execute an intelligent mobility operation at an access network level rather centralized. The presented fully DMM architecture (FDMM) is implemented for integrated WLAN and WiMAX networks, where the entire mobility functions are distributed at access level in order to achieve a fast exchange of signaling information during handover operation. In addition, IEEE 802.21 media independent handover $(M I H)$ standard is also adopted in the proposed work to execute seamless handover procedures across a heterogeneous background. The performance analysis of MIH-FDMM is compared with existing centralized mobility management (CMM) scheme in terms of handover delay, throughput, packet loss, etc. for real time traffic classes (constant and variable bit rate $(C V B R))$. Simulation result proves that the combined MIH-FDMM significantly minimize the handover delay, packet loss ratio and thus accomplishes seamless service for both real time and non-real time QoS efficiency.
\end{abstract}

Keywords: Mobile IP, Distributed mobility, Dynamic anchoring, Vertical handover, Signaling overhead, Internet traffic

\section{Introduction}

The current internet core architecture is not proficient to support advent applications over heterogeneous networks at reasonable costs. Multimedia mobile devices are generating massive amount of different applications over these networks. The existing mobility management architecture is centralized and lacks to manage mobile internet data traffic locally. Hence the IETF working group provides a dynamic DMM solution for the mobile node (MN) changes its point of attachment across the interdomain environment. The basic idea of DMM is to bring the mobility anchor closer to the $\mathrm{MN}$ in order to assist the fast exchange of signaling information during handover. Several related studies have been proposed in DMM for handover optimization based on different mobility protocols. In the first, [1] analyzes the limitations of the existing CMM approach in terms of scalability, path optimization, mobility support, etc. Later [2] proposed a possible DMM architectures at mobile core, access network and client level. The issues and different approaches are discussed to prove the efficiency of the scheme. The host based dual stack MIPv6 (DSMIPv6) as discussed in [3] achieves seamless connectivity, but not suited for mobile internet traffic which demands high speed connectivity across heterogeneous networks. The MIPv6 based DMM is discussed in [4] with DNS extension for network entities and signaling operations. Another scheme 
proposed in [5], which brings the dynamic mobility anchor closer to the MN for best service connection during handover.

Later network based DMM solution for partial approach is discussed in [6] for handover optimization. Lots of efforts have been carried out by the IETF working group for DMM solution as discussed in [7]. But the implementation issue is still a challenging task. In the existing mobility protocols, (like MIPv6, PMIPv6, and HMIPv6 etc.) The mobility contexts are anchored in a centralized manner [8-10] and are not sufficient enough to provide the required QoS for mobile internet data traffic. Therefore, the proposed work concentrates towards handover optimization by distributing the mobility anchors at access network level (next hop of mobile node) in order to support excellent QoS efficiency. The MIH-FDMM is implemented based on MIPv6 protocol for integrated WLAN / WiMAX networks.

Table 1. Comparison between CMM and DMM Approaches

\begin{tabular}{|l|l|l|}
\hline \multicolumn{1}{|c|}{ Parameters } & \multicolumn{1}{c|}{ CMM (MIPv6) } & \multicolumn{1}{c|}{ DMM (MIPv6) } \\
\cline { 3 - 3 } & Fully DMM (FDMM) \\
\hline Route Optimization & Non optimal routing & Provide best optimum routing \\
\hline $\begin{array}{l}\text { Network } \\
\text { Architecture }\end{array}$ & Hierarchical and centralized & Dynamic and distributed \\
\hline Scalability & Not scalable & More scalable \\
\hline Dynamic Mobility & $\begin{array}{l}\text { Lack of dynamic mobility } \\
\text { support }\end{array}$ & $\begin{array}{l}\text { Dynamic mobility support for } \\
\text { the user actual needs }\end{array}$ \\
\hline Reliability & $\begin{array}{l}\text { Not reliable - single point of } \\
\text { failure }\end{array}$ & $\begin{array}{l}\text { Reliable with distributed } \\
\text { anchors }\end{array}$ \\
\hline Security & $\begin{array}{l}\text { Less secure with centralized } \\
\text { scheme }\end{array}$ & Secure distributed anchoring \\
\hline $\begin{array}{l}\text { Signaling } \\
\text { Overheads }\end{array}$ & Increases & Minimum \\
\hline
\end{tabular}

The combined approach assists intelligent handover procedure and fast exchange of signaling information during handover, thus achieves excellent QoS support for CVBR traffic classes. It is proved that the MIH-FDMM scheme outperforms the existing CMM in terms of handover delay, packet dropped ratio, end-to-end delay and throughput. The rest of this paper is organized as follows. Section 2 provides the comparison, motivation and approaches of DMM schemes. Our presented approach for MIPv6 based MIHFDMM is provided in section 3. The implementation details are analyzed in Section 4, and finally conclude with the results and discussions.

\section{CMM vs DMM: Comparison, Motivation and Approaches}

DMM is an emerging approach for intelligent mobility management towards mobile internet data traffic. In the existing CMM scheme, the mobility, intelligence is focused on single end and lacks support the required QoS for all registered MN. This stimulates DMM approach to its well applicability with distributed mobility anchoring at network access level [11, 12]. The comparison of CMM and DMM schemes are listed in Table 1. The limitations of CMM are analyzed and employed in DMM for better resource utilization and to reduce the network cost. Three main DMM approaches are considered by the IETF: MIPv6, PMIPv6 and Routing based solution are discussed in [13 -15]. The MIPv6 and PMIPv6 are hosted and network based tunneling protocols whereas routing based DMM is a routing protocol (BGP) to support mobility management. In MIPv6 based DMM, the MN involves mobility signaling either in a centralized (PDMM) or distributed (FDMM) manner during handover operations. In PMIPv6 based DMM, mobility anchors provide mobility signaling without the involvement of $\mathrm{MN}$, whereas in 
routing based DMM, the route reflector mechanism is used to update and retrieve mobility options at the access level. In this paper, an MIH procedure is combined in FDMM scheme based on the MIPv6 protocol to support intelligent dynamic anchoring for seamless handover, which is extremely well suited for designing the next generation network.

\section{Analyzing MIH-FDMM in WLAN / WiMAX Integrated Architectures}

The client based MIH-FDMM scheme is presented in this section for efficient handover optimization across heterogeneous networks. Two different networks (IEEE 802. 11 and IEEE 802.16) are integrated for a fully distributed solution based on MIPv6 mobility management protocol with IP backbone.

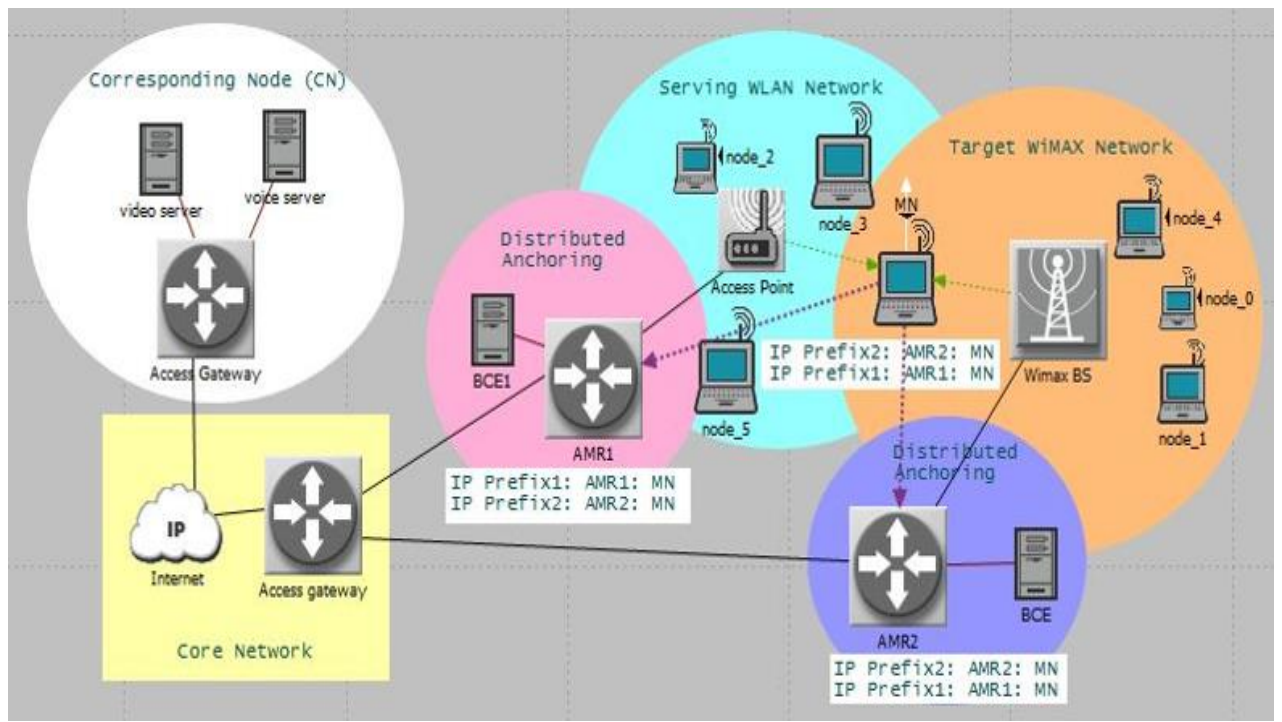

Figure 1. MIH Assisted Handover for Mipv6 Based FDMM (MIH-FDMM)

The architecture consists of $\mathrm{CN}$ (CN1-Voice application, CN2 -Video application), WLAN and WiMAX point of attachment (PoA) connected to its access mobility anchor (AMR1 and AMR2) with multiple MN and the mobility trajectory (random direction) is created in $\mathrm{MN}$ as shown in Figure 1. It is assumed that the MN initially resides in a WLAN network (home network) and moves towards WiMAX network due to poor support for mobile internet real time traffic and signal degradation in the serving network. The MIH functionalities are installed in MN and WLAN, WiMAX PoA. The detailed MIH procedure for efficient handover optimization is discussed in [16, 17].

The important unit of MIH standard is the MIH function (MIHF), which resides across MIH user (MIHU) and lower link layer device interface. The MIH standard provides three important services for efficient handover process, namely media independent event service (MIES), media independent command service (MICS) and media independent information service (MIIS). It supports three important triggers based on the link quality, MIH_Link_Up, MIH_Link_Down, MIH_Link_Going_Down events. These triggers are sufficient enough to make intelligent handover across heterogeneous networks. The MIH procedures are combined in a FDMM scheme to utilize intelligent and seamless handover operation. The entire mobility functions are distributed locally at the access network level. Thus the data and control plane purely consists of AMR, a front end for MN which performs mobility function for the MN (IP prefix allocation, local binding management and tunneling). 


\subsection{Registration Phase}

At the initial phase, the MN attached to WLAN network (receives Link_UP event from WLAN PoA) and requesting an IP prefix at AMR1 through router solicitation (RS) message which includes MN identity (MN-ID). If the service authorization is successful (enquire in AAA server), creates a local binding cache entry (BCE) for MN states and reserves IP prefix using dynamic host configuration protocol (prefix1: MN-ID) and responses through the router advertisement (RA) message. The MN then configures its new IP prefix1 at AMR1 and finally communicates with CN1- VoIP application. Therefore the time taken for the MN to complete its registration process at home network is expressed in equation (1):

$$
\mathrm{t}_{\text {reg }}^{\mathrm{AMR} 1}=\mathrm{t}_{\mathrm{RS}}+2 \mathrm{t}_{\mathrm{AAA}-\mathrm{MIIS}}\left(\mathrm{t}_{\text {req }}, \mathrm{t}_{\mathrm{res}}\right)+\mathrm{t}_{\mathrm{BCE}}^{\text {prefix1 }}+\mathrm{t}_{\mathrm{RA}}+\mathrm{t}_{\mathrm{MN}-\mathrm{conf}}
$$

The $\mathrm{MN}$ registration time is associated with router solicitation $t_{\mathrm{RS}}, \mathrm{MN}$ authorization process in AAA server $t_{A A A-M I I S}\left(t_{\text {req }}, t_{\text {res }}\right)$ with request $t_{\text {req }}$ and response time $t_{\text {res }}$, IP prefix reservation in $B C E t_{B C E}$, router advertisement $t_{R A}$ and finally $M N$ configuration time $\mathrm{t}_{\mathrm{MN}-\text { conf }}$.

\subsection{Handover to Candidate WiMAX Network (AMR1 to AMR2)}

At a later time $t$, the MN moves in a random direction, experiences Link_Down event periodically from the serving network (wishes to carry out the ongoing session anchored at AMR1 to CN1) and initiates the need for handover. At the same time, the MN receives Link_Up events from the candidate WiMAX network (WiMAX also identifies the MN attachment at AMR2). The MN enquires the upper MIIS server for neighbor WiMAX network accessibility. If it is successful, the MN request service authorization at AMR2 through RS message. The AMR2 authenticate with AAA server and finally allocates IP prefix 2 for $\mathrm{MN}$ through $\mathrm{RA}$. Then the MN demands its serving network for handover commitment, if it is granted, the MN configures another prefix 2 and sends binding update (BU) to AMR2 for registration indicating its previous state (prefix 1:AMR1). The AMR2 updates its BCE and establish a bidirectional tunnel between AMR1 and AMR2 through the exchange of access binding update (ABU) and access binding acknowledgement (ABA) messages. After receiving the binding acknowledgement (BA success registration) from AMR2, the MN waits for time to trigger to handover i.e Link_Going_Down event. If the Link_Going_Down event is triggered, the MN immediately executes handover (soft handover) to WiMAX network and continues its ongoing session with CN1. 


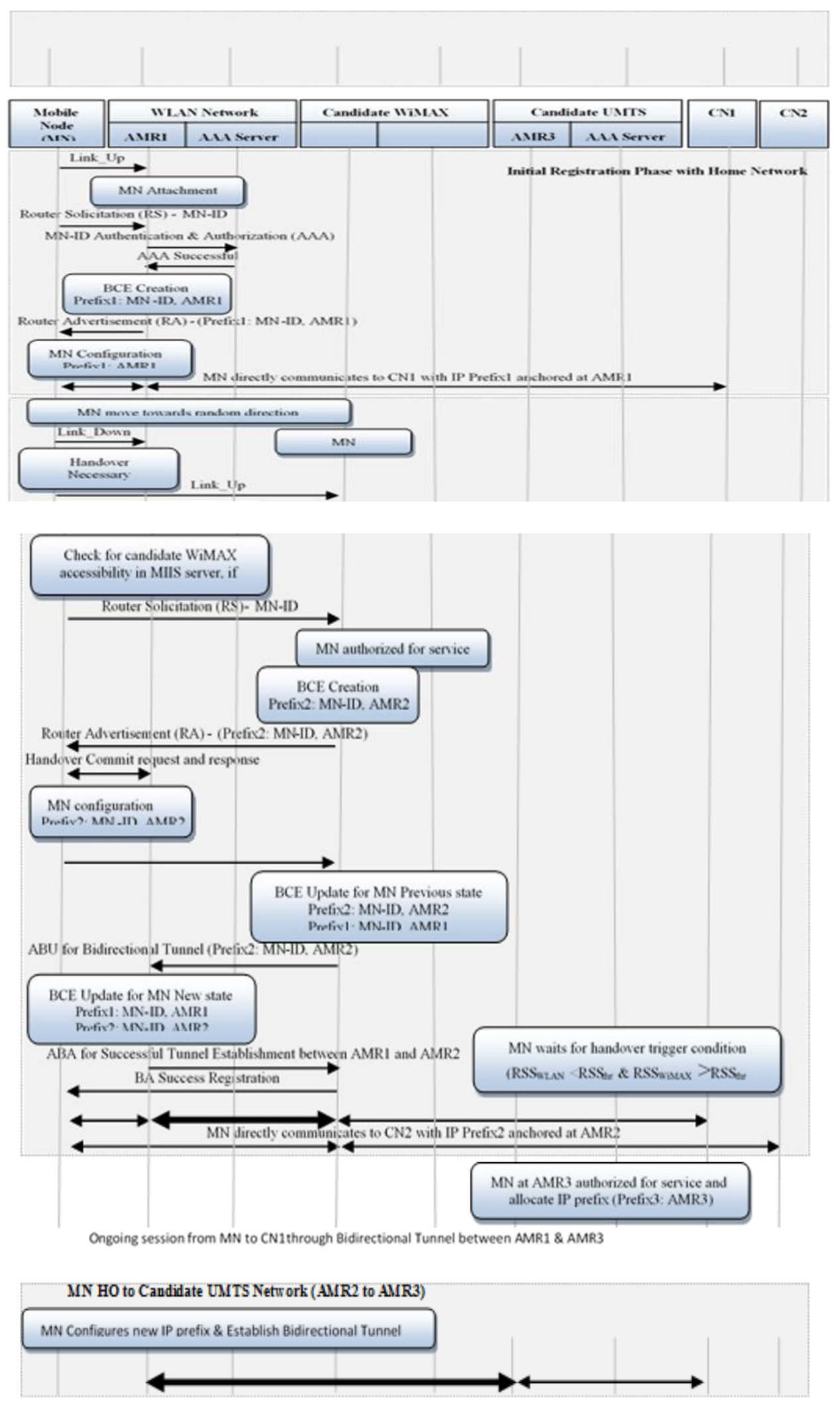

Figure 2. MIH-FDMM Handover Signaling Procedure 
The new IP prefix2 anchored at AMR2 is used for the new communication session with CN2 (Video Application - MPEG4). The vertical handover delay (VHO) from AMR1 to AMR2 is given in equation (2) - (5):

$$
\begin{gathered}
\tau_{\mathrm{VHO}}^{\mathrm{F}-\mathrm{DMM}}=\tau_{\mathrm{reg}}^{\mathrm{AMR} 2}+\tau_{\mathrm{MN}-\mathrm{BM}}+\tau_{\text {tunnel }}^{\mathrm{AMR} 2-\mathrm{AMR} 1} \\
\tau_{\mathrm{reg}}^{\mathrm{AMR} 2}=\mathrm{t}_{\mathrm{RS}}^{\mathrm{AMR} 2}+2 \mathrm{t}_{\mathrm{AAA}-\mathrm{MIIS}}^{\mathrm{AMR2}}\left(\mathrm{t}_{\mathrm{req}}, \mathrm{t}_{\mathrm{res}}\right)+\mathrm{t}_{\mathrm{BCE}}^{\text {prefix2 }}+\mathrm{t}_{\mathrm{RA}}+\mathrm{t}_{\mathrm{MN}-\text { conf }}
\end{gathered}
$$

The MN VHO delay depends on registration delay $\left(\tau_{\text {reg }}^{\mathrm{AMR} 2}\right)$, binding management (BM) delay $\left(\tau_{\text {MN-BM }}\right)$ and tunneling delay from AMR2 to AMR1 $\left(\tau_{\text {tunnel }}^{\text {AMR2-AMR1 }}\right)$. The $\mathrm{BM}$ delay is the time taken for the exchange of $t_{\mathrm{BU}-\text { req }}^{\text {reg }}$ and $\mathrm{t}_{\mathrm{BA}-\mathrm{res}}^{\mathrm{reg}}$ messages between MN and AMR2 respectively. The tunneling delay is directly proportional to the exchange of $\mathrm{ABU}\left(\mathrm{t}_{\mathrm{ABU}-\mathrm{AMR}}^{\mathrm{AMR}}\right)$ and $\mathrm{ABA}\left(\mathrm{t}_{\mathrm{ABA}}^{\mathrm{AMR} 1}\right)$ res $)$ messages between $\mathrm{AMR} 2$ and AMR1.

$$
\begin{aligned}
& \tau_{\mathrm{MN}-\mathrm{BM}}=\mathrm{d}_{\mathrm{MN}-\mathrm{AMR} 2}\left(\mathrm{t}_{\mathrm{BU}-\mathrm{req}}^{\mathrm{reg}}+\Delta_{\mathrm{BCE}}^{\mathrm{AMR} 2}\right)+\mathrm{d}_{\mathrm{tunnel}}^{\mathrm{AMR} 2-\mathrm{AMR} 1}+\mathrm{d}_{\mathrm{AMR} 2-\mathrm{MN}}\left(\mathrm{t}_{\mathrm{BA}-\mathrm{res}}^{\mathrm{reg}}\right) \\
& \tau_{\text {tunnel }}^{\mathrm{AMR} 2-\mathrm{AMR} 1}=\mathrm{d}_{\mathrm{MMAR2}-\mathrm{MMAR} 1}^{\mathrm{tunnel}}\left(\mathrm{t}_{\mathrm{ABU}-\mathrm{req}}^{\mathrm{AMR} 2}+\Delta_{\mathrm{BCE}}^{\mathrm{AMR} 1}+\mathrm{t}_{\mathrm{ABA}-\mathrm{res}}^{\mathrm{AMR}}\right)
\end{aligned}
$$

\subsection{Handover from WiMAX to Third Network (AMR2 to AMR3)}

The MN again chooses a random direction of travel towards third network (say UMTS). The handover procedure is similar to the previous case. If the service authentication is granted at AMR3, it allocates prefix 3 for MN. The MN configures third new prefix3 and sends BU to register the MN previous state at AMR2 (prefix2: AMR2). The AMR3 updates its $\mathrm{BCE}$ and sends $\mathrm{ABU}$ message to AMR2 for bidirectional tunnel establishment. The BCE is updated in AMR2 and sends ABU message to the parent AMR 1 for MN current PoA. The AMR1 updates MN new state and sends ABA directly to AMR3 for the success tunnel between AMR1 and AMR3. Finally the MN continues its ongoing session with $\mathrm{CN} 1$ after receiving BA from AMR3. The detailed operation of MIH-FDMM is illustrated in Figure 2. The MIH-FDMM avoids non optimal paths and signaling overhead during handover operation. This leads to minimum handover delay and limit packet loss ratio, which in turn improves the session continuity that really demands for delay sensitive CVBR application.

\section{Simulation Results and Discussion}

This section examines the presented vertical handover performance across heterogeneous environments based on combined MIH-FDMM approach. The scenario is implemented in OPNET software with the integration of two networks, WLAN / WiMAX overlay model. The WLAN infrastructural mode is considered in our analysis with transmission power of $0.005 \mathrm{~W}, 11 \mathrm{Mbp}$ data rate and $20 \mathrm{MHz}$ bandwidth. The WiMAX network supports medium mobility and provides best QoS for CVBR mobile internet traffic. The MN's are placed in a random position and moves with a velocity of $20 \mathrm{~m} / \mathrm{s}$ from WLAN to WiMAX network. The proposed MIH-FDMM is compared with an existing CMM scheme in terms of handover delay, throughput, end to end delay, packet loss ratio, and signaling overheads for real time CVBR traffic classes (Voice and Video). The simulation parameters for MIH-FDMM are listed in Table 2. The performance analysis is described from the following results. Figure 3(a) describes the vertical handover delay between existing CMM and proposed MIH-FDMM approaches based on MIPv6 protocol. 
Table 2. Simulation Parameters

\begin{tabular}{|l|l|l|l|}
\hline \multicolumn{1}{|c|}{$\begin{array}{c}\text { Parameters (MN } \\
\text { Configuration) }\end{array}$} & \multicolumn{1}{|c|}{ Value } & \multicolumn{1}{|c|}{$\begin{array}{c}\text { Parameters (AP } \\
\text { Configuration) }\end{array}$} & \multicolumn{1}{|c|}{ Value } \\
\hline MN Trajectory & Vector & Max. SS nodes & 100 \\
\hline Route Optimization & Enabled & Maximum Queue size & 4 \\
\hline Traffic Characteristics & $\begin{array}{l}\text { Interactive Voice, } \\
\text { Multimedia }\end{array}$ & $\begin{array}{l}\text { Receiver Power } \\
\text { tolerance }\end{array}$ & $\begin{array}{l}\text { Min (-110), } \\
\text { Max (-60) }\end{array}$ \\
\hline Antenna Gain & $-1 \mathrm{dBi}$ & $\begin{array}{l}\text { Handover ranging } \\
\text { codes }\end{array}$ & 8 \\
\hline $\begin{array}{l}\text { Max. Transmission } \\
\text { Power }\end{array}$ & $0.5 \mathrm{~W}$ & $\begin{array}{l}\text { Neighbour } \\
\text { Advertisement }\end{array}$ & $\begin{array}{l}\text { Every 10 } \\
\text { frames }\end{array}$ \\
\hline Physical Characteristics & OFDMA, 20MHz & Resource retain time & $2(200 \mathrm{ms)}$ \\
\hline BS MAC Address & Distance based & $\begin{array}{l}\text { Bandwidth request } \\
\text { start }\end{array}$ & 2 \\
\hline Max. Handover Request & 6 & Bandwidth request end & 4 \\
\hline $\begin{array}{l}\text { Handover threshold } \\
\text { hysteresis }\end{array}$ & 0.4 & No. of Transmitters & SISO \\
\hline $\begin{array}{l}\text { Max. Handover } \\
\text { Attempts }\end{array}$ & 3 & Channel quality & $4 / 16$ \\
\hline
\end{tabular}

It is observed that the vertical handover delay is larger for CMM $(0.08 \mathrm{sec})$ and minimum for MIH-FDMM (0.05 sec) scheme. Since in MIH-FDMM, the handover to candidate network is calculated in advance (since the proposed work follows $\mathrm{MIH}$ procedure) when Link_Down event is triggered from the serving network and also the mobility anchors are distributed at access level i.e. closer to the MN (FDMM) rather centralized (as in CMM). This reduces the overall signaling delay (time taken for the exchange of signaling information) between $\mathrm{MN}$ and mobility anchor during handover operation. The handover to candidate network is executed, when Link_Going_ Down event is triggered.

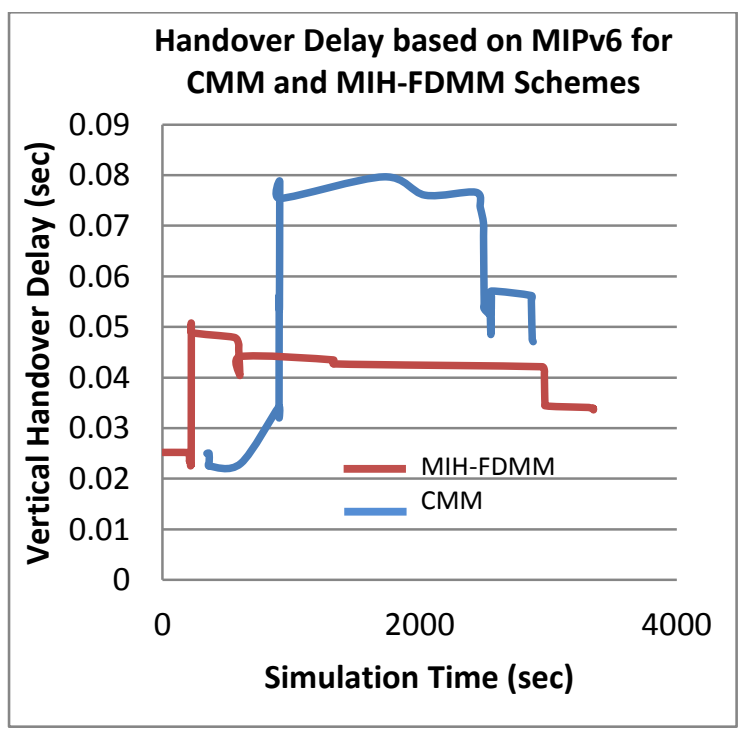

(a) 


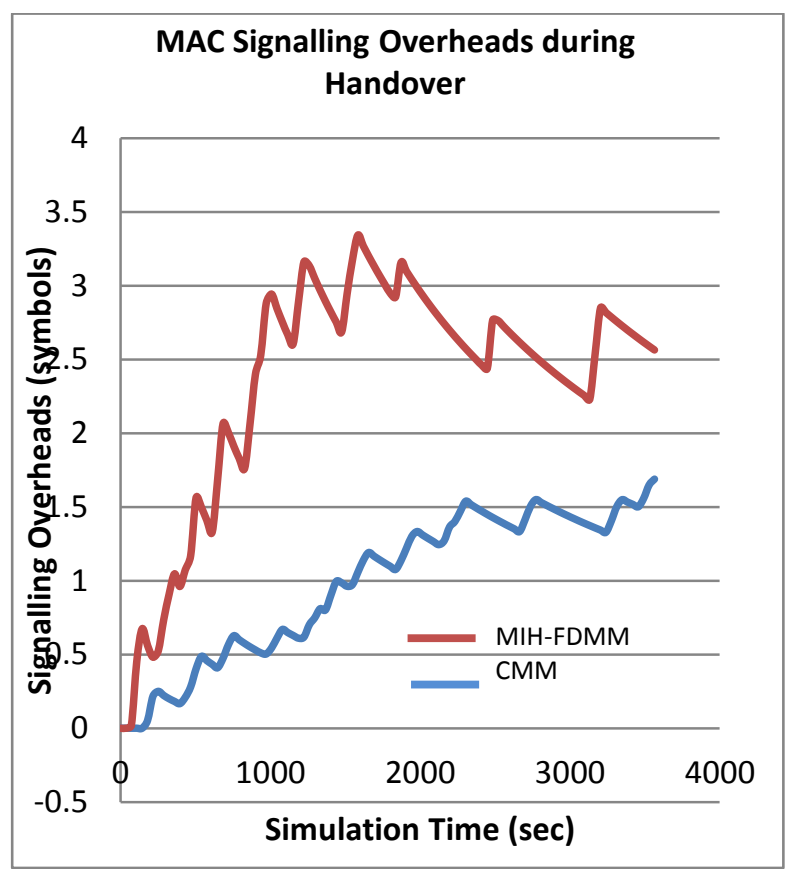

(b)

Figure 3. (a) Vertical Handover Delay, (b) MAC Signalling Overheads

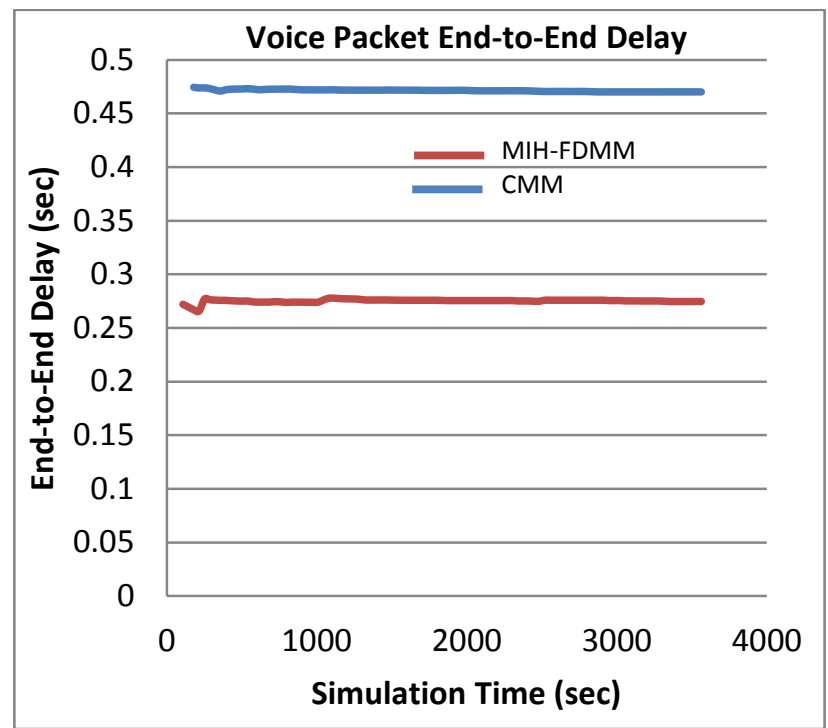




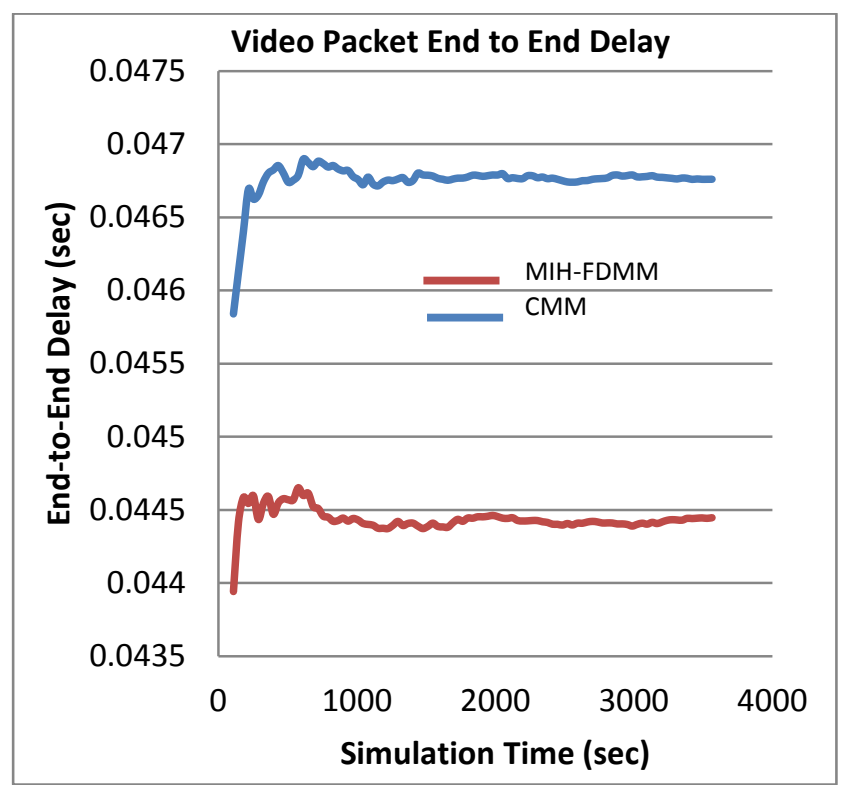

Figure 4. Voice and Video Packet End-to-End Delay

The exchange of signaling information for MIH-FDMM is maximized (3.4 symbols) in order to compute best optimized path when compared to CMM (1.5 symbols) as in Figure 3(b). The handover delay directly related to the packet loss ratio, which in turn affects the system throughput. Larger packet dropped ratio experiences a service disruption, particularly for CVBR delay sensitive applications (VoIP and Video).

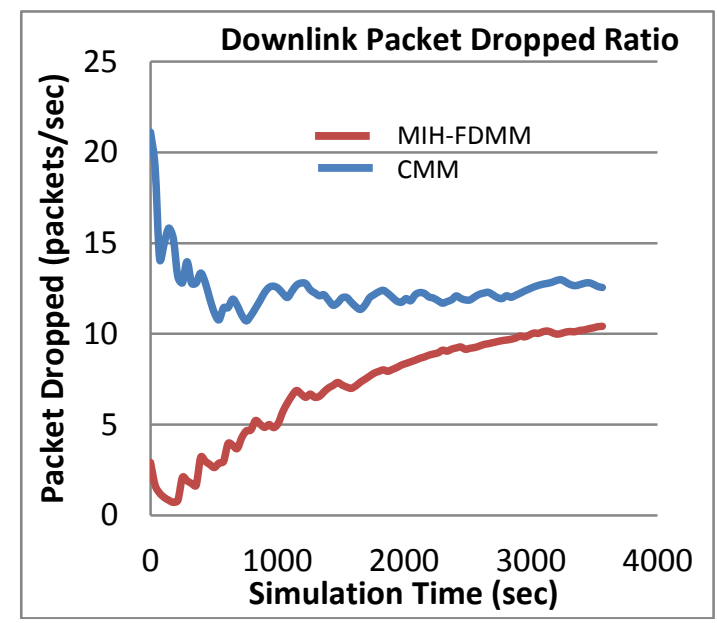

(a) 


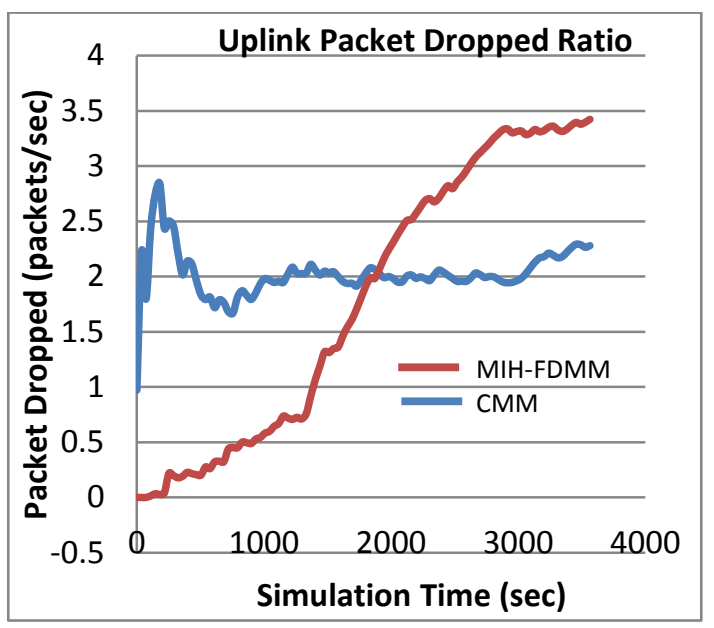

(b)

Figure 5. (a) Downlink Packet Dropped, (b) Uplink Packet Dropped

End-to-End delay and packet dropped ratio are directly proportional to handover latency. Fig. 4 and Fig. 5 clearly depict the loss ratio during handover process. It is observed that the MIH-FDMM scheme provides lesser End-to-End delay of $0.27 \mathrm{Sec}$ (voice) and $0.0445 \mathrm{Sec}$ (video), which can be tolerated and compensated with suitable decoders. Similarly the number of packet dropped ratios (uplink and downlink) for the presented scheme is very low when compared to CMM. It is noted that the CMM involves more time to execute handover process due to centralized mobility anchors. Since the time taken for the exchange of signaling information for the MN BU and tunnel establishment is maximum.

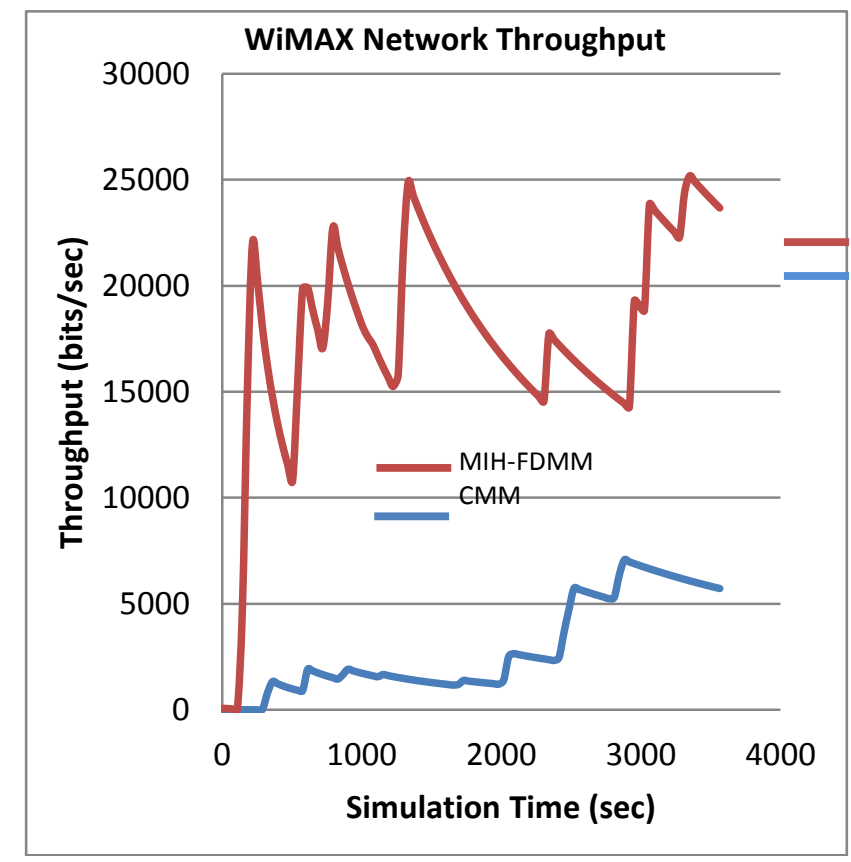

(a) 


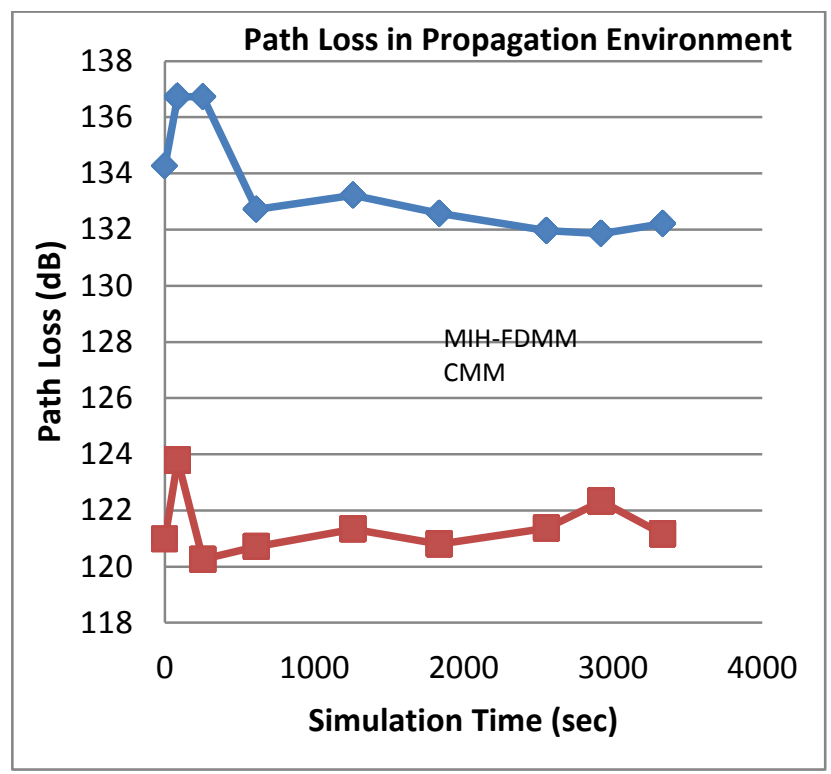

(b)

Figure 6. (a) WiMAX Throughput, (b) Propagation Path Loss

After the handover process, traffic flows through the WiMAX network by balancing both the network load and improves the system throughput as depicted in Figure 7(b). The neighbor node advertisement received during the handover process depends on the propagation path loss and mobility of MN in a wireless environment. The MIH-FDMM scheme achieves less path loss of $124 \mathrm{~dB}$, which in turn efficiently receives the neighbor advertisement with satisfied uplink and downlink signal-to-noise (SNR) ratio as shown in Figure 6 and Figure 7.

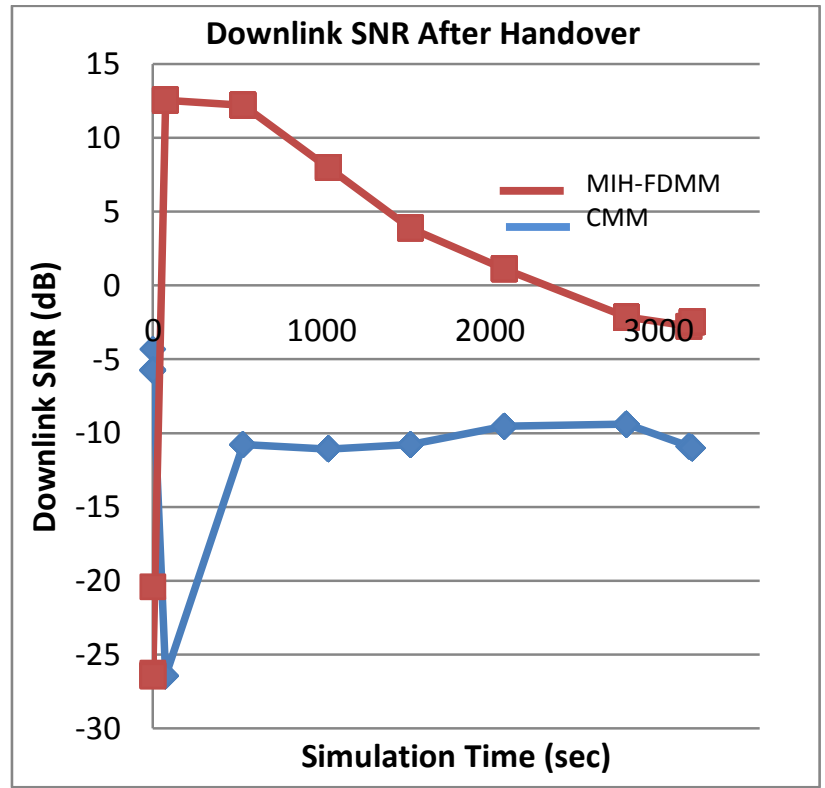

(a) 


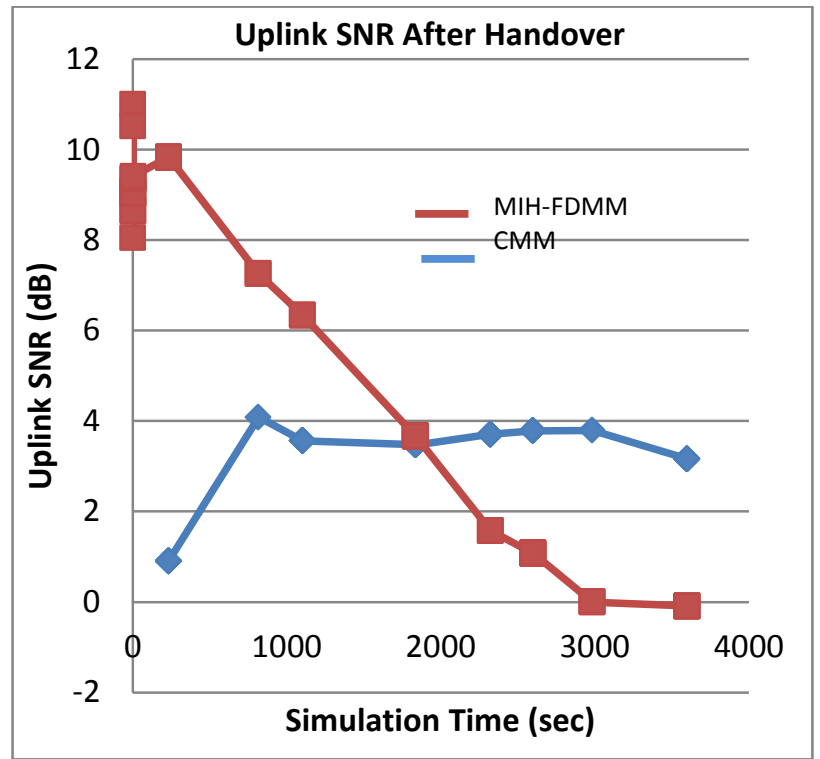

(b)

Figure 7. (a) Downlink SNR (b) Uplink SNR

Table 3 shows the comparative analysis of performance parameters of integrated WLAN and WiMAX network based on CMM and MIH-FDMM schemes. From the results and discussions, it is proved that the presented solution highly supports seamless connectivity across heterogeneous networks. Proper handover signaling achieves less handover delay, packets dropped ratio and retransmission attempts. Hence the implemented work proves to be more efficient for both delay sensitive (real time) and delay tolerant (non-real time) QoS applications.

Table 3. Comparitive analysis of CMM and MIH-FDMM

\begin{tabular}{|l|c|c|}
\hline \multicolumn{1}{|c|}{ Parameters } & CMM & MIH-FDMM \\
\hline Video traffic receiver (bytes/Sec) & 700,000 & 700,000 \\
\hline Video packet end-to-end delay (Sec) & 0.047 & 0.0445 \\
\hline Video packet delay variation & 0.0006 & 0.00025 \\
\hline Voice packet MOS value & 1.1 & 2.1 \\
\hline Voice - MOS de-jitter loss rate & 0.065 & 0.0025 \\
\hline Voice - MOS network loss rate & 0.755 & 0.55 \\
\hline Voice jitter (sec) & 0.0014 & 0.000 \\
\hline Voice packet end-to-end delay (sec) & 0.47 & 0.27 \\
\hline Voice packet delay variation & 0.00015 & 0.0015 \\
\hline Voice traffic sent (bytes/sec) & 15,000 & 75,000 \\
\hline Vertical handover delay (sec) & 0.08 & 0.05 \\
\hline RIP traffic received (bits/sec) & 1,100 & 620 \\
\hline WiMAX network throughput (bits/sec) & 6,000 & 25,000 \\
\hline WiMAX delay (sec) & 0.01 & 0.014 \\
\hline WiMAX traffic received (bits/sec) & 8,000 & 24,000 \\
\hline WiMAX queuing delay (sec) & 0.013 & 0.015 \\
\hline WiMAX periodic ranging activity & 0.05 & 0.47 \\
\hline Serving BS-ID & 3.7 & 4.2 \\
\hline Neighbour node advt. received (bits/sec) & 8,000 & 18,000 \\
\hline Downlink BLER & 0.5 & 0.4 \\
\hline Quantized CQI SNR (dB) & 18 & 24 \\
\hline Propagation path loss (dB) & 137 & 124 \\
\hline
\end{tabular}




\section{Conclusion}

Distributed mobility management is an advent paradigm for efficient mobility management in all IP flat architecture to cope mobile internet traffic locally. The MIH assisted DMM approach for fully distributed solution (FDMM) based on the MIPv6 protocol are carried out and compared with an existing CMM scheme in order to optimize handover procedures across Inter-domain environment. The presented method proves to be energy efficient with a number of distributed mobility anchors and avoids a single point of failure for the attackers. The MIH-FDMM approach is analysed in terms of handover delay, packet dropped ratio, traffic end-to-end delay, and throughput. The qualitative results suggest that MIH-FDMM considerably minimizes the handover delay to 50\% when compared to CMM scheme and hence FDMM is well suited for both delay sensitive and delay tolerant applications. Another attractive feature of our approach is that it maintains fast and seamless connectivity, outperforms the existing CMM, which really required for future generation networks.

\section{References}

[1] D. Liu, JC. Zuniga, P. Seite, H. Chan and CJ. Bernardos, "Distributed Mobility Management: Current practices and gap analysis", IETF Internet-Draft, draft-ietf-dmm-best-practices-gap-analysis, vol. 3, (2014).

[2] H.A. Chan, H. Yokota, J. Xie, P. Seite and D. Liu, "Distributed Mobility Management in Mobile Internet: Current Approaches and Issues", International Journal of Communications, vol. 6, no. 1, (2011), pp. 4-15.

[3] H. Soliman, "Mobile IPv6 Support for Dual Stack Hosts and Routers", IETF RFC 5555, (2009).

[4] S. Yan, C. Jiayin and C. Shanzhi, "A Mobile IPv6 based Distributed Mobility Management Mechanism of Mobile Internet", Proceedings of International Conference on Solid State Devices and Materials Science, Physics Procedia, vol. 25, (2012), pp. 2249-2256.

[5] P. Bertin, S. Bonjour and J.-M.Bonnin "A Distributed Dynamic Mobility Management Scheme Designed for Flat IP Architectures", New Technologies, Mobility and Security, (2008).

[6] J.-G. Ki, K.-T. Lee and D.-H. Kim, "Modeling and Simulation of Partially Distributed Mobility Management Scheme", International Journal of Multimedia and Ubiquitous Engineering, vol 9, no. 8, (2014), pp. 125-136.

[7] D. Liu, Z. Cao, P. Seite and H. Chan, "Distributed mobility management", Draft liu distributed mobility, vol. 02, (2010).

[8] C. Perkins, "IP Mobility Support for IPv4", IETF RFC 5944, (2010).

[9] S. Gundavelli, K. Leung, V. Devarapalli, K. Chowdhury and B. Patil, "Proxy Mobile IPv6", IETF RFC 5213, (2008).

[10] H. Soliman, C. Castelluccia, K. El Malki and L. Bellier, "Hierarchical Mobile IPv6 Mobility Management (HMIPv6)", RFC 4140, (2005).

[11] B. Sarikaya, "Distributed Mobile IPv6", IETF Internet-Draft, draft-sarikaya-dmm-dmipv6-00, (2012).

[12] CJ. Bernardos, A. de la Oliva and F. Giust, "A PMIPv6 solution for Distributed Mobility Management", IETF Internet-Draft, draft-bernardos-dmm-pmip-03, (2014).

[13] D.-H. Shin, D. Moses, M. Vengatachalam and S. Bagchi, "Distributed Mobility Management for Efficient Video Delivery over AII-IP Mobile Networks: Competing Approaches”, International Journal of IEEE network, (2013).

[14] J.-H. Lee, J.-M. Bonnin, P. Seite and H.A. Chan, "Distributed IP Mobility Management from the Perspective of the IETF: Motivation, Requirements, Approaches, Comparison and Challenges", International Journal of IEEE Wireless Communication, (2013), pp. 159-168.

[15] J.-H. Lee, J.-M. Bonnin and X. Lagrange, "Host Based Distributed Mobility Management Support Protocol for IPv6 Mobile Networks", Proceedings of IEEE Conference on Wireless and Mobile Computing, Networking and Communication, (2012), pp. 61-68.

[16] R. Tamijetchelvy and G. Sivaradje, "An optimal vertical handover for heterogeneous networks based on IEEE 802.21 MIH standards", Proceedings of Fifth IEEE International Conference on Advanced Computing (ICoAC), (2013), pp. 446-451.

[17] R. Tamijetchelvy and G. Sivaradje, "Analysis of IEEE 802.21 media independent handover with mobility management protocols for handover optimization", Computers and Electrical Engineering, vol. 48, (2015), pp. 119-134. 
International Journal of Future Generation Communication and Networking Vol. 9, No. 3 (2016) 